\title{
An Improved Detection Method of STATCOM Compensating Reference Current
}

\author{
LI Shengqing,YUAN Li,TANG Qi,BAI Jianxiang \\ Hunan University of Technology \\ College of Electrical and Informati on Engineering \\ Zhuzhou, China \\ e-mail: 1sq1961@sohu.com
}

\begin{abstract}
With the continuous development of power electronics technology, static synchronous compensator (STATCOM) structure increasingly multiplex. When the load current check point and the compensation injection point of STATCOM in different position and exist the difference of the phase and amplitude, which can produce a difference of detection.To solve this problem, this paper proposes an improved STATCOM compensation instruction current detection method based on $i_{p}-i_{q}$ algorithm. This method can eliminate the error and improve the detection accuracy by setting the preset phase angle and proportional integral (PI) controller according to the difference of voltage phase and amplitude between the check point and the compensation point, thus breaking the limitation that traditional $i_{p}-i_{q}$ algorithm is only applicable in the case of that the detecting point and the compensation point in the same position, which improve the accuracy of detection. Theoretical analysis and simulation results show the effectiveness of the proposed method in this paper.
\end{abstract}

Keywords-STATCOM;detection difference;compens-ation instruction current;ip-iq algorithm;preset p-hase angle

\section{INTRODUCTION}

In recent years, the harmonic pollution in the modern power grid is becoming increasingly serious which leading to the stable operation of power system is affected ${ }^{[1]}$. In order to improve the power quality problem more accurately and effectively, people has been proposed various compensation device. Among them, STATCOM can be real-time dynamic compensation of power grids automatically which is representative of the latest technology in today's compensation field ${ }^{[2,3]}$. STATCOM can dynamically tracking compensation quickly and accurately depending on the detection method of command current, so people proposed various detection methods. The harmonic current detection method based on the adaptive interference canceling theory has the detection ability of adaptive tracking, but the dynamic response is slow and exist a delay ${ }^{[4]}$. The harmonic current detection method based on the neural network method and the wavelet transform method has high detection precision, but it has computational complexity and workload shortcomings ${ }^{[5,6]}$. The harmonic current detection method based on the Fourier transform (FFT) has high detection precision and its simple to realize, but it also has computational complexity and real-time problem. The $i_{p}-i_{q}$ algorithm based on the instantaneous reactive power theory has better real-time and fast dynamic response speed, so it has been widely used in the detection of STATCOM compensation reference current ${ }^{[7,8]}$. However, the traditional $\mathrm{i}_{\mathrm{p}}-\mathrm{i}_{\mathrm{q}}$ algorithm is only applicable in the case of that the load current detecting point and the power compensation injection point of STATCOM in same location, Therefore, there are certain restrictions on the detecting of reference current for complex structure STATCOM in the engineering practice.

This paper proposed an improved ip-iq algorithm to detect STATCOM compensation reference current. This method introducing the voltage phase information of detecting point and compensation point, eliminating detection error and improving the detection accuracy by setting the phase presetting angle and PI controller, thus breaking the limitation that traditional ip-iq algorithm is only applicable in the case of that the detecting point and compensation point in the same position.

\section{TRADITIONAL $i_{p}-i_{q}$ ALGORITHM}

The instantaneous reactive power theory is based on the theorem of instantaneous active power $p$ and instantaneous imaginary power $\mathrm{q}$. With the continuous development and improvement, it proposed the $\mathrm{p}-\mathrm{q}$ and $\mathrm{i}_{\mathrm{p}}-\mathrm{i}_{\mathrm{q}}$ algorithm based on the instantaneous reactive power theory. Among them, The ip-iq algorithm has smaller error and wider application in the detection of STATCOM compensation reference current[9], and the schematic diagram shown in Fig .1.

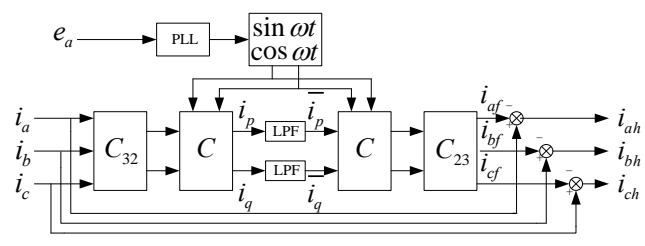

Figure 1. The schematic diagram of $i_{p}-i_{q}$ detection method

Hypothesis three phase is symmetric, the detected current is as follows[10]: 


$$
\begin{aligned}
& i_{a}=\sqrt{2} \sum_{n=1}^{\infty} I_{n} \sin \left(n \omega t+\varphi_{n}\right) \\
& i_{b}=\sqrt{2} \sum_{n=1}^{\infty} I_{n}\left[n\left(\omega t-\frac{2 \pi}{3}\right)+\varphi_{n}\right] \\
& i_{c}=\sqrt{2} \sum_{n=1}^{\infty} I_{n}\left[n\left(\omega t+\frac{2 \pi}{3}\right)+\varphi_{n}\right]
\end{aligned}
$$

Where $\omega$ is angular frequency, In and $\varphi_{n}$ are valid values and initial phase angle for each current.

The detected three phase signal go through the Clark transform $\mathrm{C} 32$ and the Park transform $\mathrm{C}$ to get the instantaneous active current ip and instantaneous reactive current iq respectively as follows

$$
\left[\begin{array}{l}
i_{p} \\
i_{q}
\end{array}\right]=C \cdot C_{32} \cdot\left[\begin{array}{l}
i_{a} \\
i_{b} \\
i_{c}
\end{array}\right]=\sqrt{3}\left[\begin{array}{l}
\sum_{n=1}^{\infty} I_{n} \cos \left[(1 \mathrm{~m} n) \omega t \mathrm{~m} \varphi_{n}\right] \\
\sum_{n=1}^{\infty} \pm I_{n} \sin \left[(1-n) \omega t-\varphi_{n}\right]
\end{array}\right]
$$

$$
\text { where } C=\left[\begin{array}{cc}
\sin \omega t & -\cos \omega t \\
-\cos \omega t & -\sin \omega t
\end{array}\right], \quad C_{32}=\sqrt{\frac{2}{3}}\left[\begin{array}{ccc}
1 & -\frac{1}{2} & -\frac{1}{2} \\
0 & \frac{\sqrt{3}}{2} & -\frac{\sqrt{3}}{2}
\end{array}\right]
$$

After through the low pass filter (LPF),ip、iq turn into $\overline{i_{p}} 、 \overline{i_{q}}$. The fundamental current can be obtained through the inverse transform as follows

$$
\left[\begin{array}{c}
i_{a f} \\
i_{b f} \\
i_{c f}
\end{array}\right]=C_{23} \cdot C \cdot\left[\begin{array}{c}
\overline{i_{p}} \\
\overline{i_{q}}
\end{array}\right]=\left[\begin{array}{c}
\sqrt{2} I_{1} \sin \left(\omega t+\varphi_{1}\right) \\
\sqrt{2} I_{1} \sin \left(\omega t-\frac{2 \pi}{3}+\varphi_{1}\right) \\
\sqrt{2} I_{1} \sin \left(\omega t+\frac{2 \pi}{3}+\varphi_{1}\right)
\end{array}\right]
$$

where $C_{23}=C_{32}^{T}, i_{a f}, i_{b f}, i_{c f}$ is the fundamental current of $i_{a}, i_{b}, i_{c}$ respectively.

Three phase current signal minus the fundamental current signal can get harmonic current, disconnect the iq gallery can get the sum of harmonic current and fundamental reactive current, reactive current can be obtained by inverse transformation. The resulting current signal obtained as compensating reference current of STATCOM, it can realize the compensation of load.

However, the traditional ip-iq algorithm only with the load voltage detecting point as the signal generator, requiring load detecting points and compensation injection point in the same voltage level, so it has certain restrictions of STATCOM compensation position in the engineering practice.

III. Improvement of STATCOM compensating reference current detection method

With multiplex structure of STATCOM, when the load current detection point and STATCOM compensation injection point located on different voltage levels, the voltage phase and amplitude between the detection point and the compensation point will have a certain deviation. However, the traditional ip-iq algorithm is only applicable to the situation that detection point and compensation points at the same voltage level and the amount of information has larger deviations, which can not be directly used for compensation. This paper analysis the principle of the instantaneous reactive power theory and presents an improved detection method. The method is based on the traditional ip-iq algorithm, by introducing the voltage information eaveb from the detection point and compensation point, there are two aspects have been improved: The one aspect is to eliminate the detection error caused by the phase difference by setting the preset phase angle, the other is to eliminate the detection error caused by amplitude differences by setting PID controller. The principle is shown in Fig .2.

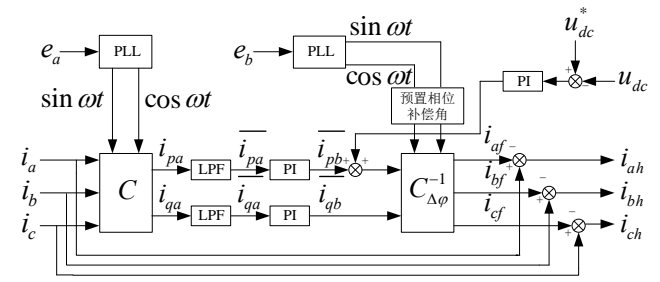

Figure 2. The principle diagram of the improved detection methods

(1) According to the difference between the load detection point voltage ea and compensation injection point voltage $\mathrm{e}_{\mathrm{b}}$, setting a preset phase angle in the detection circuit, so as to eliminate the error caused by the phase difference detection. While the compensation point voltage $e_{b}$ and detection point voltage $e_{a}$ at different phase, it can't directly to occurs the phase of $\mathrm{e}_{\mathrm{a}}$ as a inversion transform signal. Here set the initial phase angle of detection point voltage is 0 , the difference of voltage initial phase angle between the detection and compensation point is $\Delta \varphi$, the transformation matrix $\mathrm{C}$ is as follow

$C=\sqrt{\frac{2}{3}}\left[\begin{array}{ccc}\sin (\omega t) & \sin \left(\omega t-\frac{2 \pi}{3}\right) & \sin \left(\omega t+\frac{2 \pi}{3}\right) \\ -\cos (\omega t) & -\cos \left(\omega t-\frac{2 \pi}{3}\right) & -\cos \left(\omega t+\frac{2 \pi}{3}\right)\end{array}\right]$

The phase of $e_{b}$ occurs as a signal of inverse transformation and introducing a preset phase angle $\Delta \varphi$ before the inverse transformation matrix $C_{\Delta \varphi}^{-1}$. The inverse transformation matrix $C_{\Delta \varphi}^{-1}$ is obtained as follow

$$
C_{\Delta \varphi}^{-1}=\sqrt{\frac{2}{3}}\left[\begin{array}{cc}
\sin (\omega t+\Delta \varphi) & -\cos (\omega t+\Delta \varphi) \\
\sin \left(\omega t+\Delta \varphi-\frac{2 \pi}{3}\right) & -\cos \left(\omega t+\Delta \varphi-\frac{2 \pi}{3}\right) \\
\sin \left(\omega t+\Delta \varphi+\frac{2 \pi}{3}\right) & -\cos \left(\omega t+\Delta \varphi+\frac{2 \pi}{3}\right)
\end{array}\right]
$$

After the improvement, the fundamental component of the DC component through the inverse transform matrix obtained from the $C_{\Delta \varphi}^{-1}$ without error, so as to improve the accuracy of detection.

(2) Increase the PI controller after the low pass filter 
(LPF), use the voltage amplitude ratio between the $e_{a}$ and the $e_{b}$ as the adjustment coefficient of ratio control $P$ to reduce the steady-state error. The PI controller can eliminate the static error of variables, its dynamic performance and steady state performance of anti-jamming is good. When the traditional ip-iq algorithm in detecting current, he current after through the transformation matrix $\mathrm{C}$ can be calculated as followed

$$
\left[\begin{array}{l}
i_{p a} \\
i_{q a}
\end{array}\right]=C\left[\begin{array}{l}
i_{a} \\
i_{b} \\
i_{c}
\end{array}\right]
$$

Then through a low-pass filter to get the fundamental current signal $\overline{i_{p a}}, \overline{i_{q a}}$.The measurement point and the compensation point has voltage amplitude difference, in order to ensure the power balance, inversion of fundamental current can not be replaced directly. The power balance equation is as follow

$$
\frac{3}{2} U_{a m} I_{a m}=\frac{3}{2} U_{b m} I_{b m}
$$

Where $U_{a m} 、 U_{b m}$ is the voltage amplitude of the measurement point and the compensation point respectively, $I_{a m} 、 I_{b m}$ is the Current amplitude of the measurement point and the compensation point respectively.

Setting the amplitude ratio $\mathrm{K}_{\text {up }}$ between the measuring point voltage $e_{a}$ and the compensation point voltage eb as follow

$$
K_{u p}=\frac{U_{b m}}{U_{a m}}
$$

According to formula (7)、(8), can obtain the relationship as follow

$$
I_{b m}=\frac{1}{K_{u p}} I_{a m}
$$

where $1 / \mathrm{K}_{\mathrm{up}}$ is proportional control factor. The DC component $\overline{i_{p a}}, \overline{i_{q a}}$ to the PI regulator by proportional resonance operation can obtained $\overline{i_{p b}} 、 \overline{i_{q b}}$ respectively, And then sent to an inverse transform part, can get the fundamental current as

$$
\left[\begin{array}{l}
i_{a f} \\
i_{b f} \\
i_{c f}
\end{array}\right]=C_{\Delta \varphi}^{-1}\left[\begin{array}{l}
\overline{i_{p b}} \\
\overline{i_{q b}}
\end{array}\right]
$$

In order to make STATCOM work normally, here introduce a voltage balancing controller. to ensure $u_{d c}$ constant. The difference value between the $u_{d c}$ and reference voltage $u_{d c}^{*}$ through a PI controller, then the value and $\overline{i_{p b}}$ superposition to maintain effect of the voltage stability.

\section{SIMULATION AND EXPERIMENTAL RESULTS}

In this paper, the STATCOM simulation model has built by using the Matlab/simulink, which verified the correctness and effectiveness of the improved detection method. The simulation model consists of the main circuit of STATCOM 、 signal detection circuit, , control circuit and drive circuit. The simulation parameters are as follows: the power line voltage is $311 \mathrm{~V}$, power line current is $32 \mathrm{~A}$, power grid frequency is $50 \mathrm{~Hz}$, the load resistance is $100 \Omega$, the load inductance is $2 \mathrm{mH}$, the circuit resistance was $5 \Omega$, line inductance is $2 \mathrm{mH}$.

Fig .3 (a)、(b) is the waveform of power supply voltage and load current respectively, it can be clearly seen that voltage magnitude of load imbalance and serious distortion of current waveform. Fig .4 (a)、(b) is the a phase instruction current waveform of traditional ip-iq algorithm and the method proposed in this paper respectively. Through the contrast can be seen obviously, current waveform of traditional ip-iq algorithm is poor. The improved detection method proposed in this paper has good real-time and proves that the improved method can realize the accurate detection of compensation current.

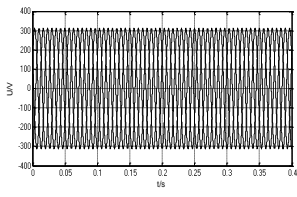

(a) power supply voltage

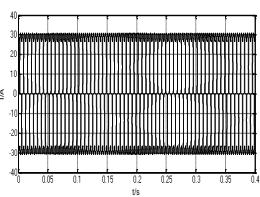

(b) load current
Figure 3 . The waveform of power supply voltage and load current

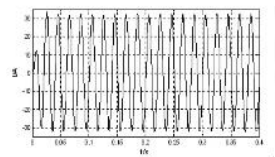

(a) The traditional method

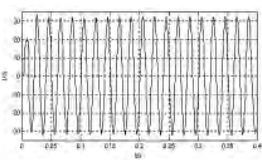

(b) The improved method
Figure 4. Reference current of $a$ phase

The method proposed in this paper apply to the experiment in the STATCOM system, the results are shown in Fig .5、6. Fig .5 is the grid current waveform before compensation, the distortion is serious. Fig .6 (a)、 (b) is the grid current waveform after compensation. Starting from $0.04 \mathrm{~s}$ to compensate the power grid, it can be seen clearly that the effect of grid current waveform compensating by traditional algorithm is poor and contains a lot of harmonic. The grid current waveform after compensation obtained by the method proposed in this paper has been quite close to the sine wave, the harmonic content is reduced from the original $4.3 \%$ to $1.2 \%$, which verifies the feasibility and effectiveness of the proposed method.

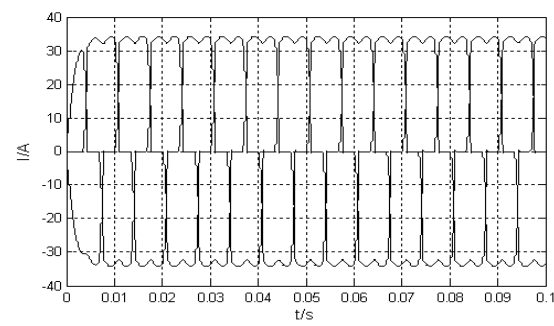

Figure 5. The grid current waveform before compensation 


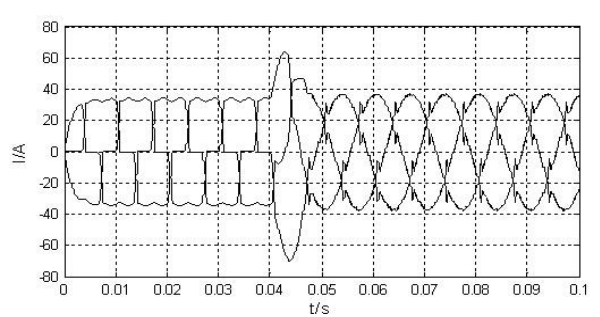

(a)The traditional method

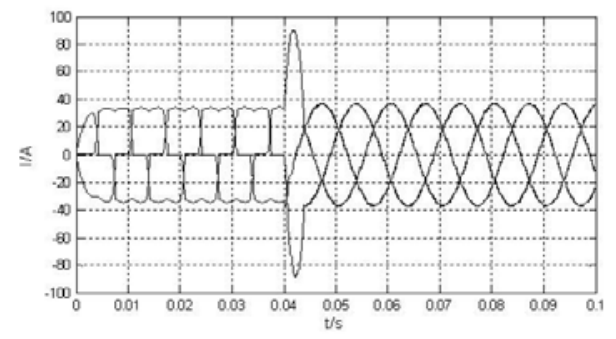

(b) The improved method

Figure 6. The grid current waveform after compensation

\section{Conclusions}

This paper is based on the instantaneous reactive power theory and in-depth analysis of the basic principle of the $i_{p}-i_{q}$ algorithm, it points out that the limitations of this method in the detection of STATCOM instruction current and proposes an improved instruction current detection method, which sets a phase compensation angle and add the PI controller after the low pass filter to solve the instruction current detection problem in the case of the STATCOM detection point and the compensation point in different position. Simulation experiment is carried out through the analysis of the traditional $\mathrm{i}_{\mathrm{p}}-\mathrm{i}_{\mathrm{q}}$ algorithm and the improved method, the results shows that the improved method can detect compensation instruction current better and has more extensive scope of application.

\section{ACKNOWLEDGMENT}

Hunan Natural Science Foundation of China (2015JJ 5009);Key Discipline Constrution Project of Hunan Provi nce,China (201176); Key Laboratory of Hunan Colleges and Universities,China (2014249); Hunan Science and Technology Innovation Team Project of Colleges and Universities,China(2014207).

\section{REFERENCES}

[1] Jiang Zhonghua, Luo An, Zhao Wei, Wu Fang-ci. A simulation system of real-time power quality monitoring and harmonic suppression for distribution network. Power System Technology, 2010,34(8): 18-34.

[2] Luo Chenglian, Ji Yong, Liu Zunyi. The principle and the implementation of static synchronous compensator (STATCOM). China electric power press, 2005.

[3] Li Shengqing, Xu Wenxiang. DC capacitor voltage balancing control for cascade STATCOM in Wind Farm. Transaction Of China Electrotechnical Society, 2013, 28(3): 248-253.

[4] Xia Xiangyang, Luo An. Novel method for measuring harmonic current based on adaptive frequency-tracking. High Voltage Engineering, 2008, 34(8): 1715-1719.

[5] Zeng Lingquan, Bai Zhiliang, Zeng Dejun. Harmonic detection based on adaptive neural network for active power filter. Electric Power Automation Equipment, 2010, 30(2): 33-37.

[6] Zhou Lin, Xia Xue, Wan Yunjie. Harmonic measurement of three-phase asymmetrical system based on wavelet transform. High Voltage Engineering, 2007, 33(3): 109-112.

[7] Chang Xianrong, Wang Huiyun, Zhang Haisheng. A novel method to detect reference compensation current for DSTATCOM. Power System Technology, 2013, 37(10): 2819-2824.

[8] Zhou Lin, Meng Jing, Liu Qiang. Detecting methods of harmonics and reactive current based on instantaneous reactive power theory. High Voltage Engineering, 2009, 35(2): 385-392.

[9] Akagi H, Kananzawa Y, Nabae A, Generalized theory of the instantaneous reactive power in three-puase circuits[C]//IEEE\&JIEE.Proceedings IPEC. Tokyo:1983,103(7): 483-490.

[10] Wang Zhaoan, Yang Jun, Liu Jinjun. Harmonic suppression and reactive power compensation. Mechanical industry press, 2005 